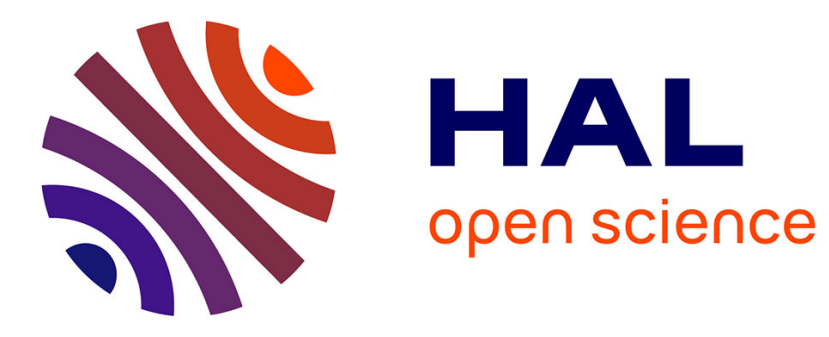

\title{
Prices of Robustness and Reblending in Oil Industry
}

\author{
Stefan Janaqi, Jorge Aguilera, Mèriam Chèbre
}

\section{To cite this version:}

Stefan Janaqi, Jorge Aguilera, Mèriam Chèbre. Prices of Robustness and Reblending in Oil Industry. IFAC - Manufacturing Modelling, Management, and Control, Jun 2013, Saint Petersbourg, Russia. pp.180-185, 10.3182/20130619-3-RU-3018.00023 . hal-00853648

\section{HAL Id: hal-00853648 https://hal.science/hal-00853648}

Submitted on 19 Sep 2013

HAL is a multi-disciplinary open access archive for the deposit and dissemination of scientific research documents, whether they are published or not. The documents may come from teaching and research institutions in France or abroad, or from public or private research centers.
L'archive ouverte pluridisciplinaire HAL, est destinée au dépôt et à la diffusion de documents scientifiques de niveau recherche, publiés ou non, émanant des établissements d'enseignement et de recherche français ou étrangers, des laboratoires publics ou privés. 


\title{
Prices of robustness and reblending in oil industry
}

\author{
Stefan Janaqi.* Jorge Aguilera*. Meriam Chébre** \\ *LGI2P Ecole des Mines d'Alès, Parc Scientifique Georges Besse, 30035 Nîmes Cedex 1, France \\ (Tel:00 334 66387005; e-mail: stefan.janaqi@mines-ales.fr). \\ **Advanced Process Control Department, Technical Direction, TOTAL Refining \&Marketing, Le Havre, France (e-mail: \\ meriam.chebre@total.com)
}

\begin{abstract}
In this paper we present a method to calculate the prices of robustness and reblending through a robust real-time optimization method for the on-line linear oil blending process. Our approach places this problem in a wider frame where different sources of uncertainty inherent to the blending process appear. The polytopal structure of our problem permits a robust approach that is simpler than the classical theory of Ben-Tal and Nemirovskii which needs convex programming tools. Our method is intended to avoid reblending and we measure its performance in terms of the blend quality giveaway and feedstocks prices. The difference between the nominal and the robust optimal values (the price of robustness) provides a benchmark for the cost of reblending which is difficult to estimate in practice. This new information can be used to adjust the level of conservatism in the model. Additional critical information for the control system is produced.
\end{abstract}

Keywords: Robustness, Blending, Polyhedra, RTO, Linear Programming.

\section{INTRODUCTION}

The oil blending process consists in determining the optimal proportions to blend from a set of available components such that the final product obtained fulfills a set of specifications on their properties. The legal constraints and environmental considerations make it harder nowadays to obtain the "good" blend. When the blend does not fulfill the constraints, it has to be reblended and this is a loss of money, time and energy. So, we address the problem of evaluating the price of reblending and how to avoid it.

A blending system is typically constituted by three functional subsystems: the scheduling subsystem, the on-line optimizer and the control subsystem. The scheduling subsystem is the one in charge of the general refinery production planning. The scheduling subsystem uses a linear program to calculate for a given period the recipes $u$, properties $y$ and volumes $V$ of reference for a sequence of blends $\left(u_{k}^{0}, y_{k}^{0}, V_{k}^{0}\right), k=$ $1, \ldots, K$ (typically $K \in[10,15])$. These calculations are based on the mean characteristics of components. Several sources of uncertainty (see below) perturb the process. The online optimizer is then required to update the target recipe which may became sub-optimal, or even infeasible, due to these disturbances in the process. For the first blends of the sequence, the online process fits well with the forecasted $\left(u_{k}^{0}, y_{k}^{0}, V_{k}^{0}\right)$. Nevertheless, after a number of blends, it happens that the blending environment differs significantly from the mean characteristics considered by the scheduling subsystem. In these cases, it is very difficult for the online optimizer to take the right decision. The feedback is based on measures of the blends' and components' properties gathered by online analyzers. Finally, the control subsystem is in charge to adjust the component's flow rates (the recipe $u$ ) in order to attain the current target recipe. Here we focus on the Real Time Optimization (RTO) system formed by the online optimizer and the control subsystem. An RTO loop consist of calculating the optimal recipe $u^{*}$ for the new constraints and adjusting the actual recipe $u$ by $u^{*}-u$. Typically, our control subsystem adjusts the recipe every 5 minutes by calling the online optimizer with different sets of constraints (up to 100 calls in 5 minutes). A RTO is one that meets this time constraint. This motivates our approach for robust blend models that can be calculated efficiently. Our uncertainty model gives rise to linear models that are efficiently solved.

An important characteristic in the oil blending process is the strict requirements over some properties controlled by environmental, legal and technological standards. If these properties are not satisfied, one can correct the blend to a certain limit by dumping the appropriate additives in it. Otherwise, the blend must be reblended. The additives are too expensive whereas reblending reduces the refinery capacity, so one should consider these blends' bounds as hard constraints which must be satisfied.

On the other hand, oil blending is a complex process where several unknown and uncertain factors affect the blend's properties. In addition to the plantmodel error produced by the linearization of blending laws, there are other sources of uncertainty: measure errors on components and properties caused by instruments' precision; uncertain knowledge of the components' properties due to upstream process variations and uncontrolled blending conditions such as air temperature, humidity, etc. All these are typical uncertainties arising in any RTO problem (see Zhang 2001).

Hard constraints on the blends' properties combined to these sources of uncertainty are the ideal characteristics to apply the Robust Optimization (RO) techniques (see Ben-Tal \& al 2009). A main issue in the RO techniques is to consider a deterministic and set-based uncertainty model. No probabilistic assumption is made over the uncertainty and the solution obtained is optimal for any realization of the 
uncertainty in a given set. RO techniques seem to be ad hoc to address an uncertain RTO problem where the feasibility is the primary concern.

Some previous works have been devoted to find robust solutions to the blending problem. In (Hendrix 1996), a geometric approach is presented for the product and mixture design problems but only considering the uncertainty due to measurement imprecision. To deal with components' properties uncertainty, (Singh 2000) proposed a non-linear blend RTO system based on predictions of feedstocks properties whereas (Wang \& al 2007) presented a chance constraint model and a hybrid: neural networks - genetic algorithm solution. More recently, (Chèbre \& al 2010) introduced a linear blending control algorithm which handles this type of uncertainties via an estimator of the components' properties. A more general method based on stochastic programming which covers various types of uncertainty is presented in (Zhang \& al 2002).

Our approach begins by highlighting the polytopes underlying the blending process. This and the special form of uncertainty on components (see below) permit to model the robustness without need of convex programming tools. Linear programming solver is all we need. This point is of greatest interest in the real time processes under investigation. Our models permit to calculate a number of critical informations such as: the relative price of reblending, the price of robustness, the interval of feasible blend volumes, etc.

In this paper we study the blending process with linear blending laws and the components stocked in running tanks. The paper's structure follows. Section 2 presents the Blending Polytopes inherent to the Blending Problem and the Basic Equation which will be used to introduce the updated data in the RTO model. Section 3 analyzes the general case when the heel of a previous blend is used in the production of the new blend and a RTO approach is proposed to solve the Blending Problem. In Section 4 we construct uncertainty sets for various types of uncertainty. These sets determine the robust regions for the blending polytopes in which the new robust RTO method is based. Finally, the price of robustness and reblending is introduced and explained through a real numerical example in Section 5.

\section{BLENDING POLYTOPES}

Let's denote by $n$ and $m$ the number of components and properties respectively. We will represent a blend's recipe by a vector $u \in R^{n}$ such that $u_{j}$ is the percentage of component $j$ present in the blend whereas the blend's properties will be denoted by a vector $y \in R^{m}$. Then the set of components is described by the $m \times n$ matrix $B$ where, $B_{i, j}$ is the $i^{\text {th }}$ physical and / or chemical characteristic of the component $j$. It is customary when blending to use the heel of a previous blend to produce a new one. The process starts then with a volume $V_{0}$ of a previous blend with properties $b_{0} \in R^{m}$ and continues by adding gradually a volume $V$ of the new blend to obtain a final product's volume $V_{\text {total }}=V_{0}+V$.

We suppose that blend's properties are linear combinations of components' properties. In general they are not, but a number of transformations exist that give good linear approximations of them. So, for any $v \in[0, V]$ the blend's properties corresponding to the recipe $u$ are determined by the basic equation:

$$
y(u)=\alpha_{v} \cdot b_{0}+\beta_{v} \cdot B \cdot u
$$

where, $\alpha_{v}=\frac{V_{0}}{V_{0}+v}, \beta_{v}=\frac{v}{V_{0}+v}$.

Having a blend's volume $V_{0}$ with properties $b_{0}$, equation (1) can be used at any time in the process to plan the production of a fixed volume $v \in[0, V]$. We just update $b_{0}$ and the matrix $B$ and take $V-v$ as the remaining volume to pour. For this planning horizon, the optimal recipe is obtained by solving the Blending Problem (BP):

$$
\begin{aligned}
& \min _{\mathrm{u}} c \cdot u \\
& \underline{u} \leq u \leq \bar{u} \\
& \frac{y}{} \leq y(u) \leq \bar{y} \\
& 1^{T} \cdot u=1,0 \leq u \leq 1
\end{aligned}
$$

Here $c \in R^{n}$ gives the components' costs. A recipe $u$ is subject to components availability and hydraulic bounds which impose the component's constraints (2). Similarly, the properties constraints (3) are determined by quality lower $(\underline{y})$ and upper $(\bar{y})$ bounds. Additionally, properties' bounds are labeled as hard $\left(\underline{y}_{H}, \bar{y}_{H}\right)$ or soft $\left(\underline{y}_{S}, \bar{y}_{S}\right)$. Hard bounds are related to legal, commercial and environmental specifications which must be satisfied whereas soft bounds can be violated but incurring into quality giveaway. Finally, (4) are the percentage constraints. We note $H_{1}=\left\{u \in R^{n} \mid 1^{T} \cdot u=1\right\}$ and the simplex $S_{1}=\left\{u \in R^{n} \mid 1^{T} \cdot u=1,0 \leq u \leq 1\right\}$.

Now, there are 3 distinct polytopes and one polyhedron participating in blending process. Geometrically, the intersection of the regions determined by the components constraints and $S_{1}$ defines the recipe's polytope:

$$
U=\left\{u \in S_{1} \mid \underline{u} \leq u \leq \bar{u}\right\}
$$

which represents the set of all recipes that can be produced. On the other hand, the properties constraints define the polyhedron (as it may be unbounded):

$$
C_{U}=\left\{u \in R^{n} \mid \underline{y} \leq y(u) \leq \bar{y}\right\}
$$

Both are defined in the component's space.

In properties space, the image of $U$ under the basic equation is the possible blend's polytope:

$$
P=\left\{y(u) \in R^{m} \mid u \in U\right\}
$$

Finally, the target polytope is defined by:

$$
C=\left\{y \in R^{m} \mid \underline{y} \leq y \leq \bar{y}\right\}
$$

We notice that, among the four preceding polytopes, only $P$ and $C_{U}$ depend on $v$. At this point we have identified $U, C_{U}, P, C$ underlying the BP and we observe that for a fixed volume $v$, a feasible solution of $\mathrm{BP}$ is a recipe $u \in U \cap C_{U}$ with properties $y(u) \in P \cap C$. 


\section{BLENDING FROM A PREVIOUS BLEND}

In any RTO loop, the on line optimizer is required to produce a recipe that permits the controller to guide the blending process. In Section 2 we stated that for a fixed volume $v$ to pour, the optimal recipe can be obtained by solving the BP. However, the BP can result to be infeasible because of the uncertainty pervading the process or for instance, when a previous blend with properties out of specification is used in the production of the new blend. Therefore, a method to compute at any time the best recipe for the planning horizon is required. Later we present this method but first we discuss the BP feasibility when the heel of a previous blend is used.

If the blend is produced from scratch or the volume to pour is too big compared with the heel's volume, that is, if $V_{0}=0$ or $v \rightarrow \infty$ then the basic equation reduces to $y(u)=B \cdot u$ and the polytope $P(v)$ does not depend on $V_{0}$. However, the fact of using the actual blend's properties $b_{0}$ in the basic equation to update the model inside the RTO loop, makes blending from a previous blend the regular case. Thus, we have $V_{0} \neq 0$ and $P(v)$ "moves" from $b_{0}$ toward $P(\infty)$ as $v$ increases. In properties space the polytope $C$ doesn't depend on $v$. We suppose that there is an interval of volumes $\left[V_{\text {Min }}, V_{\text {Max }}\right]$ such that $P(v) \cap C \neq \varnothing, \forall v \in\left[V_{\text {Min }}, V_{\text {Max }}\right]$. Even if $P(v)$ and $C$ may have different affine dimensions, they both lie into $R^{m}$. Consequently, this intersection makes sense. In other words, when $P(v)$ moves it encounters $C$. If this were not the case, one has to change the components in order to assure feasibility. The BP is feasible only for these volumes.

Having the values $V_{\text {Min }}$ and $V_{\text {Max }}$ helps to select the appropriate planning horizon at each RTO loop. Choosing a planning horizon by taking a volume $v<V_{\text {Min }}$ produces an infeasible BP and makes it necessary to generate an alternative recipe with a possible deterioration of the overall performance of the RTO method. On the other side, $V_{\text {Max }}$ is the maximum volume to blend when looking for a blend within specifications. After $V_{\text {Max }}$ has been poured, $P(v)$ moves away from $C$ and the blend's properties deteriorate progressively.

Furthermore, knowing $V_{\text {Min }}$ and $V_{\text {Max }}$ helps the control system to reduce unnecessary and inefficient interventions. The last ones having as purpose to correct the blend to be within specifications but often they are based on a limited view of the problem. In order to compute the interval $\left[V_{\text {Min }}, V_{\text {Max }}\right]$, let's rewrite the basic equation:

$$
y(u)=\frac{1}{1+u_{0}} \cdot\left(u_{0} \cdot b_{0}+B \cdot u\right)
$$

with $u_{0}=\frac{v_{0}}{v}$. Now we present the RTO method:

\section{Blending RTO Method}

We denote by $C_{H U}=\left\{u \in R^{n} \mid \underline{y}_{H} \leq y(u) \leq \bar{y}_{H}\right\}$ the polyhedron defined by the hard constraints and let $y_{T} \in C_{H U}$ be an "ideal" target blend without quality giveaway provided by the scheduling department.
Any RTO loop proceeds through the steps I. to VI. as follows:

I. Incorporate the new available information $\left(V_{0}, b_{0}, B, V\right)$ in the basic equation. If $V_{0}=0$, solve problems (11), (14) and (15) in this order until having a feasible solution. Otherwise, go to step II.

II. Compute $V_{\text {Min }}$ and $V_{\text {Max }}$ by solving the linear programming problem:

$\max , \min _{\mathrm{u}_{0}, \mathrm{u}} u_{0}, u \in U \cap C_{U}, u_{0} \geq 0$

If Problem (10) is infeasible or if $V \notin$ $\left[V_{\text {Min }}, V_{\text {Max }}\right]$ go to step IV. Otherwise, a blend without quality giveaway exists for any volume $v \in\left[V_{\text {Min }}, V_{\text {Max }}\right]$. Choose a blending horizon $v \in\left[V_{\text {Min }}, V_{\text {Max }}\right]$ and go to step III.

III. Compute the optimal recipe:

$$
\min _{\mathrm{u}} c \cdot u, u \in U \cap C_{U}
$$

Notice that solving Problem (11) with $v$ free and the additional constraint:

$$
u_{0 \operatorname{Min}} \leq u_{0} \leq u_{0 \operatorname{Max}}
$$

we obtain at the same time the optimal blending horizon $v^{*} \in\left[V_{\text {Min }}, V_{\text {Max }}\right]$ and the optimal recipe for this volume. This is the best choice if it's not imperative to produce a particular volume. In this case STOP. Otherwise go to step IV.

IV.Forget the components costs and focus only on the hard constraints. Compute the interval $\left[V_{\text {Min }}, V_{\text {Max }}\right]$ by solving the problem:

$\max ,(\min )_{\mathrm{u}_{0}, \mathrm{u}} u_{0}, u \in U \cap C_{H U}, u_{0} \geq 0$

If this problem is infeasible or if $V \notin$ $\left[V_{\text {Min }}, V_{\text {Max }}\right]$ go to step VI. Otherwise, go to step $\mathrm{V}$.

V. Look for a recipe $u \in U \cap C_{H U}$ and a volume $v^{*} \in\left[V_{\text {Min }}, V_{\text {Max }}\right]$ producing the blend with minimal quality giveaway. To do this, perform a dichotomic search over $v$ solving at each step (with $v$ fixed) the problem:

$$
\begin{gathered}
\min _{\mathrm{u}, \mathrm{t}}\|y(u)-y(t)\|_{1} \\
u \in U \cap C_{H U}, t \in C_{U}
\end{gathered}
$$

VI. Find $u \in U$ to produce a volume $V$ of a blend with properties as near as possible to the ideal blend $y_{T}$ :

$$
\min _{\mathrm{u}}\left\|y(u)-y_{T}\right\|_{1}, u \in U
$$


Following these steps, the RTO method always produces a recipe that guides the control process. In step I, $V_{0}=0$ and by solving problems (11), (14) and (15) we obtain the minimal cost recipe, the blend with minimal quality giveaway or the closest blend to $y_{T}$. If BP is feasible for some volume $v \in\left[V_{\text {Min }}, V_{\text {Max }}\right]$, then a blend without quality giveaway exists and the method generates the one of minimal cost (step III). Otherwise, the hard constraints become the priority and the method searches for a blend satisfying them while minimizing the blend's quality giveaway (step V). Finally, if there is no blend satisfying the hard constraints, the recipe producing the blend with properties as close as possible to $y_{T}$ is proposed (step VI).

We finish this section by stressing the possible application of the RTO method to determine the appropriate heel's volume to use in the blend. In our analysis, $V_{0}$ is considered as fixed but we can solve (11) with the additional constraint (12) with $V_{0}$ and $V$ free in order to obtain the cheapest recipe $u^{*}$ for some $u_{0}^{*} \in\left[u_{0 \text { Min }}, u_{0 \text { Max }}\right]$. Then we can find (by means of the relation $u_{0}=\frac{V_{0}}{v}$ a suitable pair $V_{0}, V$. The choice of norm $L_{1}$, in problems (14) and (15) permits to obtain blends violating a minimum number of properties. Moreover, it gives LP problems and there are a lot of well-known fast and robust LP solvers.

\section{ROBUST RTO}

In the previous section we proposed a blending RTO method based on blending polytopes and its evolution with the blended volume. Looking to reduce the model deviations produced by some uncertainties, the method uses blend's and components' properties updates to feedback the model via the basic equation. However, model updating may fail to guarantee even a feasible solution. A main reason of this failure is the implicit assumption that data remains unchanged inside each RTO loop.

For instance, when on-line blending is used, $B$ values fluctuate with time because components are issued from different process presenting also perturbations. To address this problem, (Singh 2000) proposed a blending RTO method which updates the model with predictions of the components' properties. Although this method improves the model accuracy, it continues to be non-robust as it depends on the quality of the predictions. Moreover, uncertainty in the blending process affects other factors than $B$ values as we will see below.

In accordance with (Zhang 2001), uncertainty in any RTO system may be of four types: 1. Process uncertainty: components properties, temperature, humidity, etc.; 2. Measurement uncertainty; 3. Model uncertainty; 4. Market uncertainty: components availabilities and prices, blends demands, etc. In this work we consider the uncertainties arising from components measurement and blend's properties measurement (type 2) and the uncertainty caused by imprecise knowledge of the components properties (type 1).

Measurement and components properties uncertainties manifest geometrically in different ways: for the first type, the real recipe (the real percentages of each component used in the blend) and its properties may differ from the nominal ones. So, the real recipe and its properties are located in neighborhoods of the nominal recipe and its properties respectively. For the second type, the real matrix $\tilde{B}$ differs from the nominal matrix $B$ and hence the real polytope $\tilde{C}_{U}$ is different from the nominal polytope $C_{U}$. In both cases, when a nominal feasible recipe $u \in U \cap C_{U}$ is computed, the real recipe may lie outside the polytope $C_{U}$ and the real blend's properties may be outside the polytope $C$. Then the real recipe results to be infeasible.

An intuitive idea to fix our RTO method against measure and $B$ uncertainties follows from the previous geometric information. The idea consists in computing for polytope $C_{U}$ its convex robust region $R C_{U}$ such that any point in this region resists to $B$ uncertainty and to measurement errors. That is, any point in $R C_{U}$ is guaranteed to remain inside the real polytope for all possible realizations of $B$ and any measurement error, within reasonable limits.

So, the robust RTO method will consist in replacing polytope $C_{U}$ by $R C_{U}$ in the RTO method from Section 3. To develop this idea, first we need to model and measure the uncertainty we would like to be protected of and then to compute the appropriate robust region of $C_{U}$. This development follows the Robust Optimization (RO) theory (see Ben-Tal \& al 2009).

Let $u$ be a nominal recipe and $y(u)$ its properties. To model the components and properties measurement uncertainties, we suppose that the real recipe $\tilde{u}$ lies in the ball $S(u, \delta u)$ of radius $\delta u$ and center $u$ whereas the real blend's properties $\tilde{y}$ lies in the ball:

$$
S(y(u), \delta y)=\left\{y \in R^{m} \mid y(u)-\delta y \leq y \leq y(u)+\delta y\right\}
$$

We are given the minimal $\underline{B}$ and maximal $\bar{B}$ values of $B$. In order to model matrix $B$ uncertainty, we use interval sets. That is, we suppose that each real value $\tilde{B}_{i, j}$ is comprised in the interval $I B_{i, j}=\left[B_{i, j}-\varepsilon_{i, j}^{-}, B_{i, j}+\varepsilon_{i, j}^{+}\right]$around its nominal value $B_{i, j}$ for some positive values $\varepsilon_{i, j}^{-}, \varepsilon_{i, j}^{+}$.

Here we could use different sets and any norm to model the uncertainty. The level of conservatism (how much we want to be protected of uncertainty) and the problem complexity depend on these choices. Taking interval sets and the max norm, the robust regions obtained are polytopes and the complexity in the model is preserved at the expense of being probably too conservative (we are protected from the worst deviations of all $B$ values and from the biggest measurement errors occurring all at the same time). Now we proceed to construct the robust region of polytope $C_{U}$.

Any point $u \in C_{U}$ is robust regarding $B$ uncertainty iff $\underline{y} \leq \tilde{y}(u) \leq \bar{y}$. That is, iff for any $\widetilde{B}$ such that $\widetilde{B}_{i, j} \in I B_{i, j}$,

$$
\underline{y_{i}} \leq \alpha_{v} \cdot b_{0, i}+\beta_{v} \cdot \tilde{B}_{i} \cdot u \leq \bar{y}_{i}, i=1, \ldots, m
$$

holds. Notice that any row $\widetilde{B}_{i}$ can be expressed parametrically as $\tilde{B}_{i}(z)=B_{i}+z^{-T} Q_{i}^{-}+z^{+T} Q_{i}^{+}$, with, $Q_{i}^{-}=\operatorname{diag}\left(\varepsilon_{i, 1}^{-}, \ldots, \varepsilon_{i, n}^{-}\right), Q_{i}^{+}=\operatorname{diag}\left(\varepsilon_{i, 1}^{+}, \ldots, \varepsilon_{i, n}^{+}\right), z^{-}=$ 
$\min (0, z), z^{+}=\max (0, z)$ for some $z \in R^{n}$ such that $\|z\|_{\infty} \leq 1$. Therefore we can deduce that $u \in C_{U}$ is robust regarding $B$ uncertainty iff for $i=1, \ldots, m$

$$
\underline{y_{i}}+\beta_{v} \cdot \varepsilon_{i}^{-} \cdot u \leq y_{i}(u) \leq \bar{y}_{i}-\beta_{v} \cdot \varepsilon_{i}^{+} \cdot u
$$

From now on we denote by $\gamma_{i}^{-}(u)=\beta_{v} \cdot \varepsilon_{i}^{-} \cdot u$ and $\gamma_{i}^{+}(u)=\beta_{v} \cdot \varepsilon_{i}^{+} \cdot u$.

In addition to $B$ uncertainty, $u \in C_{U}$ resists also to components' measure uncertainty if and only if any point in $S(u, \delta u)$ satisfies (17). That is, iff for $i=1, \ldots, m$

$$
\underline{y}_{i}+\gamma_{i}^{-}(u) \leq y_{i}(u+t) \leq \bar{y}_{i}-\gamma_{i}^{+}(u)
$$

holds for all $t \in R^{n}$ such that $\|t\|_{p} \leq \delta u$. Then, computing the minimum and maximum on $t$ we get that (18) holds iff

$$
\underline{y_{i}}+\gamma_{i}^{-}(u)+\delta_{i} \leq y_{i}(u+t) \leq \bar{y}_{i}-\gamma_{i}^{+}(u)-\delta_{i}
$$

with $\delta_{i}=\beta_{v} \cdot \delta u \cdot\left\|B_{i}\right\|_{q}$. This is a result from duality, $\frac{1}{p}+\frac{1}{q}=1$. With $p=1$, the problems remains linear.

Equivalently, any recipe $u \in C_{U}$ is robust regarding $B$ uncertainty and properties' measure uncertainty iff

$$
\underline{y_{i}}+\gamma_{i}^{-}(u) \leq y_{i}(u)+Z_{i} \leq \bar{y}_{i}-\gamma_{i}^{+}(u),
$$

holds for all $Z \in R^{m}$ such that $\left|Z_{i}\right| \leq \delta y_{i}$. As previously, computing the minimum and maximum on $Z$, (20) holds if

$$
\underline{y_{i}}+\gamma_{i}^{-}(u)+\delta y_{i} \leq y_{i}(u) \leq \bar{y}_{i}-\gamma_{i}^{+}(u)-\delta y_{i}
$$

Finally, letting $\Delta_{i}=\max \left(\delta_{i}, \delta y_{i}\right)$ we obtain the robust region $R C_{U}$ of polytope $C_{U}$ :

$$
\begin{gathered}
R C_{U}=\left\{u \in R^{n} \mid \underline{y}_{i}+\gamma_{i}^{-}(u)+\Delta_{i} \leq y_{i}(u) \leq \bar{y}_{i}-\gamma_{i}^{+}(u)-\right. \\
\left.\Delta_{i}, i=1, \ldots, m\right\}
\end{gathered}
$$

Any $u \in R C_{U}$ resists to $B$ uncertainty and to components and properties' measurement uncertainties.

To summarize, let's consider a nominal feasible recipe $u \in U \cap C_{U}$. If $\tilde{u} \in S(u, \delta u)$ and $\tilde{y} \in S(y(u), \delta y)$ for some $\delta u \in R^{+}$and $\delta y \in R^{m+}$ and if $B_{i, j} \in I_{i, j}$, then the recipe $\tilde{u}$ will be feasible in reality: $\tilde{u} \in \widetilde{U} \cap \tilde{C}_{U}$.

The RTO method proposed in Section 3 transforms then in a robust RTO method by a simple substitution of $C_{U}$ with $R C_{U}$. This reduces the impact in implementation as the structure of RTO remains the same. As $V$ and $V_{0}$ may be considered as free variables in the RTO method, we can describe polytope $R C_{U}$ by using explicitly (19) and (21) in association with the identity $\beta_{v}=\frac{1}{1+u_{0}}$ instead of (22) where a max is involved. This robust RTO method depends completely on the robust region $R C_{U}$ and to obtain it we only need to determine the values of $\varepsilon^{-}, \varepsilon^{+}, \delta u, \delta y$. This information is available for the class of real-world problems we are concerned with. It's worthwhile to note that while $\delta u$ and $\delta y$ are considered as fixed values independent of the RTO loop's length, $\varepsilon^{-}$and $\varepsilon^{+}$ depend on it. As fluctuations on $B$ may accumulate over time, the longest the loop's length is, the biggest these fluctuations can be.

Here we limit the analysis to measurement and components properties uncertainties. However, other types of uncertainty manifest geometrically in the same way and thus can be treated identically. For instance, when the uncertainty is due to uncontrolled factors like temperature or humidity, the real blend's properties are located in a ball around the nominal blend's properties. To model the uncertainty in the components prices, we can transform the optimization problem to one with certain objective function and such that uncertainty appears as a constraint $(c \cdot u \leq \bar{c})$. Then we can construct the uncertainty sets and determine the way it affects the robust regions. We can proceed similarly for the uncertainty in the components availabilities which affects the robust region $R U$ of polytope $U$.

\section{THE PRICE OF ROBUSTNESS AND REBLENDING}

In this section we present a real example (with scaled values) in order to illustrate and compare some key aspects of the RTO method and its robust counterpart. Here we show how to compute practically the price of robustness and reblending. The $\mathrm{BP}$ consists in producing a fixed volume $V_{\text {total }}=$ $5000 \mathrm{~m}^{3}$ of blend from 8 components and $V_{0}=2000 \mathrm{~m}^{3}$ of the heel's volume from a previous blend. Each component and the previous blend has 7 properties to be controlled during the process and they are represented by the $7 \times 8$ matrix $B$ and vector $b_{0}$ respectively. Vectors $y_{\min }$ and $y_{\max }$ stand for the properties bounds while vector $c$ denotes the components' cost.

\begin{tabular}{|l|r|r|r|r|r|r|r|r|}
\cline { 2 - 10 } \multicolumn{1}{c|}{} & \multicolumn{10}{|c|}{$B$} \\
\hline$y_{1}$ & 36.00 & 36.00 & 32.00 & 42.00 & 16.00 & 31.00 & 35.00 & 46.00 \\
\hline$y_{2}$ & 0.04 & 0.04 & 0.03 & 0.08 & 0.08 & 0.14 & 0.06 & 0.55 \\
\hline$y_{3}$ & 630.00 & 620.00 & 600.00 & 580.00 & 620.00 & 600.00 & 540.00 & 450.00 \\
\hline$y_{4}$ & 32.77 & 32.77 & 32.77 & 16.98 & 16.98 & 37.72 & 24.08 & 8.26 \\
\hline$y_{5}$ & 937.95 & 937.95 & 636.62 & 199.06 & 199.06 & 170.47 & 1381.90 & 2.80 \\
\hline$y_{6}$ & 0.80 & 0.10 & 0.05 & 0.04 & 1.50 & 2.50 & 0.05 & 0.01 \\
\hline$y_{7}$ & 50.00 & 49.00 & 50.00 & 55.00 & 25.00 & 39.00 & 41.00 & 45.00 \\
\hline$c$ & 87.06 & 87.06 & 87.02 & 86.00 & 83.08 & 78.05 & 87.06 & 117.01 \\
\hline
\end{tabular}

\begin{tabular}{|r|r|r|}
\hline$b_{0}$ & $y_{\min }$ & $y_{\max }$ \\
\hline 30.00 & 30.00 & 46.00 \\
\hline 1.66 & 0.18 & 1.66 \\
\hline 640.00 & 540.00 & 640.00 \\
\hline 35.19 & 6.98 & 35.19 \\
\hline 1381.90 & 2.02 & 432.09 \\
\hline 1.81 & 0.00 & 10.00 \\
\hline 40.00 & 40.00 & 55.00 \\
\hline
\end{tabular}

In order to produce a robust recipe, we assume that components and properties measurement errors are bounded by $\delta y=[0.12,0.0003,20,0.0826,0.028,0.000049,0.2]$ and $\delta u=0.01$ respectively. Regarding $B$ uncertainty, we dispose of $B^{-}$and $B^{+}$the absolute lower and upper bounds of matrix $B$. Let define, $T^{-}=B-B^{-}, T^{+}=B^{+}-B$ and 
$T=B^{+}-B^{-}$. So, there are $\varepsilon^{-}$and $\varepsilon^{+}$such that $B-\varepsilon^{-} \leq$ $B \leq B+\varepsilon^{+}, \quad$ with $\quad \varepsilon^{-}=\min \left(T^{-}, \theta \cdot T\right)$ and $\varepsilon^{+}=$ $\min \left(T^{+}, \theta \cdot T\right)$ for some $0<\theta<1$. As we stated in Section 4 , the values of $\delta u$ and $\delta y$ are fixed during the process whereas $\theta$ is directly related to the RTO loops' length. We take $\theta=0.01$.

We solve (10) with $V_{0}=2000 \mathrm{~m}^{3}$, and obtain $\left[u_{0 \operatorname{Min} R}, u_{0 \operatorname{Max} R}\right]=[0,0.0362]$ and the corresponding robust feasible volumes interval: $\left[V_{\text {MinR }}, V_{\text {MaxR }}\right]=[55304, \infty]$. The corresponding interval for the nominal case is $\left[V_{\text {Min }}, V_{\text {Max }}\right]=$ $[34587, \infty]$. This means that we need to produce at least $34587 \mathrm{~m}^{3}\left(55304 \mathrm{~m}^{3}\right)$ in order to get a (robust) blend within specifications which uses completely $V_{0}$. Taking only the hard constraints, we obtain similar intervals.

If we decide to produce $V_{\text {total }}=5000 \mathrm{~m}^{3}$ using $V_{0}=$ $2000 \mathrm{~m}^{3}$ then we solve Problem 15 and we obtain a recipe with cost $90.72 € / \mathrm{m}^{3}$ but the blend is out of specifications. Actually, this blend violates only one property's bound but by more than $80 \%$. Instead of this, we can compute the biggest heel's volume allowing us to produce $5000 \mathrm{~m}^{3}$ of robust blend. This is the decision taken in practice. From relations $u_{0 \operatorname{MaxR}}=\frac{V_{0}}{V}$ and $V_{\text {total }}=V_{0}+V$ we obtain $V_{0 M a x}=$ $1720 \mathrm{~m}^{3}$.

Next, fixing $V_{0}=1720 \mathrm{~m}^{3}$ and $V=4285.5 \mathrm{~m}^{3}$ we solve (11) to obtain the optimal robust recipe $u_{R}^{*}=[0.1428,0.0819,0.0352,0.1049,0.2,0.2,0.0352,0.2]$ with cost $c_{R}^{*}=90.72 € / \mathrm{m}^{3}$. Incidentally, this is the same recipe that produces the blend out of specifications! On the other hand, the optimal nominal recipe

$$
u_{N}^{*}=[0.143,0.082,0.048,0.169,0.2,0.2,0.035,0.123]
$$

has a cost of $c_{N}^{*}=88.35 € / \mathrm{m}^{3}$. Therefore, producing a robust recipe induces a cost increase of $2.68 \%$. However, we observe that if we take $V_{0}=0$ (no reblending), then the robust recipe cost is 87.70 and the nominal recipe cost is 87.04 producing a cost increase of only $0.76 \%$.

From these results we are interested in comparing the price of robustness with the reblending cost (the cost difference between the recipes obtained when the heel's volume is used and when it is not). In order to provide a fair comparison, the price of robustness is obtained by taking $V_{0}=0$ (no reblending involved) and the reblending cost from the nominal recipes (no robustness involved).

To compute the price of robustness we conduct a blending simulation over 36 RTO loops of 2-hours length. In Table 1 we show the average recipe's cost over the 36 periods and the percentage increase in cost $(\Delta c)$ from the nominal recipe to the robust recipe for different $\theta$ values and taking $V_{0}=0$.

Then we generate the optimal nominal recipes when $V_{0}=$ $168.44\left(V_{0 \text { Max }}\right.$ for $\left.\theta=0.01\right)$ and $V_{0}=0$ are used in the production of $5000 \mathrm{~m}^{3}$ of blend. The cost of these recipes are 88.29 and 87.04 respectively. We observe that for this case, the recipe's cost with reblending is $\frac{(88.29-87.04)}{87.04}=1.43 \%$ greater than the nominal recipe's cost whereas the relative price of robustness is of only $0.35 \%$ for a significant value of $\theta=0.1$.

\begin{tabular}{|c|c|c|c|}
\hline$\theta$ & $c_{R}^{*}$ & $c_{N}^{*}$ & $\Delta c(\%)$ \\
\hline 0.01 & 87.3851 & 87.1097 & 0.32 \\
\hline 0.02 & 87.3542 & 87.0763 & 0.32 \\
\hline 0.03 & 87.3847 & 87.1036 & 0.32 \\
\hline 0.04 & 87.3632 & 87.0795 & 0.32 \\
\hline 0.05 & 87.4058 & 87.1194 & 0.33 \\
\hline 0.06 & 87.4134 & 87.1249 & 0.33 \\
\hline 0.07 & 87.4232 & 87.1309 & 0.33 \\
\hline 0.08 & 87.4258 & 87.1306 & 0.34 \\
\hline 0.09 & 87.4297 & 87.1307 & 0.34 \\
\hline 0.1 & 87.4077 & 87.1062 & 0.35 \\
\hline
\end{tabular}

Table 1: Relative price of robustness for different levels of uncertainty.

\section{CONCLUSION}

The main achieving of our paper is to propose and implement a set based robustness model. Each call to our RO linear solver takes $0.25 \mathrm{~s}$ in average on a benchmark of real problems (up to 20 components and 30 properties). We thus meet the real time constraint for the online optimization. The solutions obtained with the linear models differ from the real blends less than the laboratory measure errors. The limit of our approach is the underlying form of uncertainty. Others models of uncertainty will lead to convex programming techniques that are more time consuming. Moreover, our approach doesn't apply in the case of non linear blending laws.

\section{REFERENCES}

Ben-Tal, A., Ghaoui, L. E., Nemirovski, A., (August 2009). Robust Optimization (Princeton Series in Applied Mathematics). Princeton University Press.

Chèbre, M., Cref, Y., Petit, N., (April 2010). Feedback control and optimization for the production of commercial fuels by blending. Journal of Process Control 20 (4), 441-451.

Hendrix, E., (July 1996). Finding robust solutions for product design problems. European Journal of Operational Research 92 (1), 28-36.

Singh, A., (February 2000). Model-based real-time optimization of automotive gasoline blending operations. Journal of Process Control 10 (1), 43-58.

Wang, W., Li, Z., Zhang, Q., Li, Y., (2007). On-line optimization model design of gasoline blending system under parametric uncertainty. In: Proceedings of the 15th Mediterranean Conference on Control \& Automation.

Zhang, Y., (June 2001). Results analysis for trust constrained real-time optimization. Journal of Process Control 11 (3), 329-341.

Zhang, Y., Monder, D., Fraser Forbes, J., (April 2002). Realtime optimization under parametric uncertainty: a probability constrained approach. Journal of Process Control 12 (3), 373-389. 